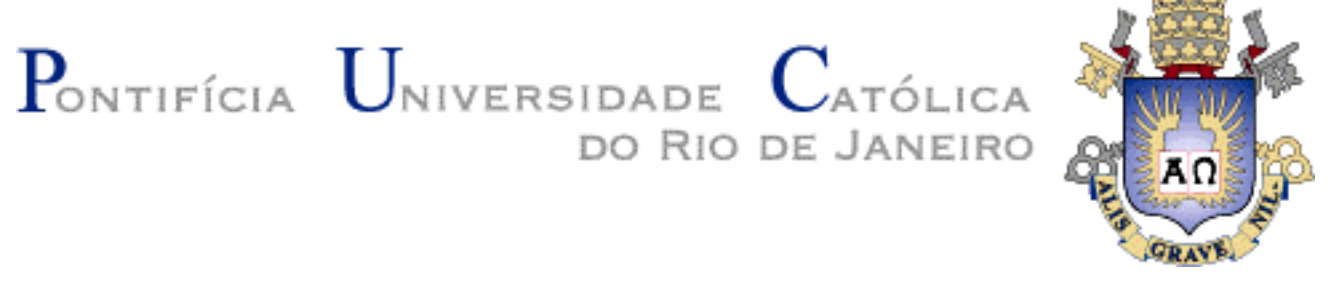

Marcelo Ferreira Moreno

Um Framework para Provisão de QoS em Sistemas Operacionais

Dissertação de Mestrado

Dissertação apresentada como requisito parcial para obtenção do grau de Mestre pelo Programa de Pósgraduação em Informática do Departamento de Informática da PUC-Rio.

Orientador: Prof. Luiz Fernando Gomes Soares

Rio de Janeiro agosto de 2002 


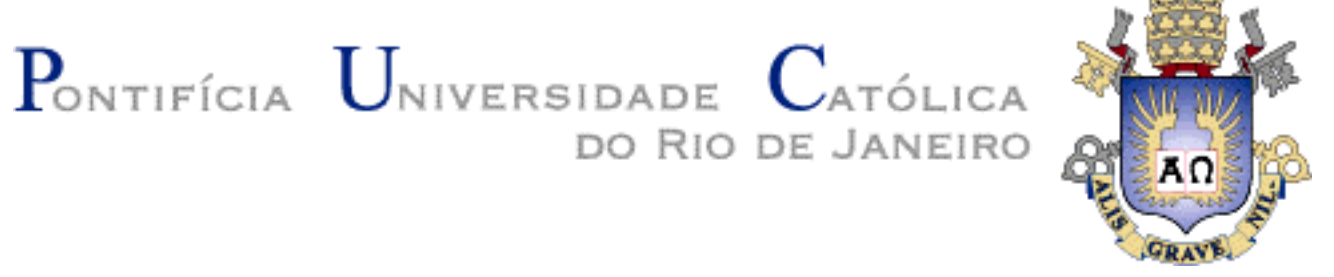

Marcelo Ferreira Moreno

\section{Um Framework para Provisão de QoS em Sistemas Operacionais}

Dissertação apresentada como requisito parcial para obtenção do grau de Mestre pelo Programa de Pósgraduação em Informática do Departamento de Informática da PUC-Rio. Aprovada pela Comissão Examinadora abaixo assinada.

Prof. Luiz Fernando Gomes Soares Orientador

Departamento de Informática - PUC-Rio

Prof. Sérgio Colcher

Departamento de Informática - PUC-Rio

Prof. Markus Endler Departamento de Informática - PUC-Rio

Prof. Ney Dumont Coordenador Setorial do Centro Técnico e Científico - PUC-Rio

Rio de Janeiro, 16 de agosto de 2002 
Todos os direitos reservados. É proibida a reprodução total ou parcial do trabalho sem autorização da universidade, do autor e do orientador.

\section{Marcelo Ferreira Moreno}

Graduado em Informática pela Universidade Federal de Viçosa, onde desenvolveu projetos na área de inteligência artificial, manipulando redes neurais. Desde dezembro de 2001, é colaborador de um projeto internacional de código livre, cujo objetivo é a criação de uma interface para a configuração dos mecanismos de qualidade de serviço presentes no sistema operacional Linux. Atualmente, integra o grupo de pesquisadores do Laboratório TeleMídia da PUC-Rio, trabalhando nas áreas de sistemas operacionais, redes de computadores e aplicações distribuídas multimídia.

Ficha Catalográfica

Moreno, Marcelo Ferreira

Um framework para provisão de QoS em sistemas operacionais / Marcelo Ferreira Moreno; orientador: Luiz Fernando Gomes Soares. - Rio de Janeiro : PUC, Departamento de Informática, 2002.

[12], 105 f. : il. ; $30 \mathrm{~cm}$

Dissertação (mestrado) - Pontifícia Universidade Católica do Rio de Janeiro, Departamento de Informática.

Inclui referências bibliográficas.

1. Informática - Teses. 2. Qualidade de Serviço. 3. Sistemas operacionais, 4. Adaptabilidade. 5. Frameworks. 6. Controle de admissão. 7. Escalonamento de recursos. I. Soares, Luiz Fernando Gomes. II. Pontifícia Universidade Católica do Rio de Janeiro. III. Título. 
Este trabalho é dedicado

A Lorenza, pela cumplicidade, respeito, carinho e amor sempre presentes em nossas vidas, unidas pela graça de Deus.

Aos meus pais, Wanderley e Jasmina, pela compreensão e incentivo em todos os momentos decisivos de minha vida, traçada por eles sobre o caminho do bem.

Aos amigos Antônio Moisés e Maria do Carmo, por me acolherem como um filho e por confiarem a mim o bem mais precioso de suas vidas.

A Deus, pela luz que me guia. 


\section{Agradecimentos}

Agradeço ao meu orientador, Professor Luiz Fernando Gomes Soares, a oportunidade, as valiosas contribuições e o emprego de sua experiência, fundamentais para a conclusão deste trabalho. Agradeço, também, a sua amizade, incentivo e ajuda pessoal, sempre presentes nos momentos difíceis dessa caminhada.

Obrigado aos colegas do Laboratório TeleMídia, pelo ambiente agradável de trabalho e pela disposição em ajudar no surgimento de dúvidas e problemas. Em especial, quero agradecer aos amigos Alésio Pfeifer e Luciana Lima, pelo companheirismo mútuo e pelos imprescindíveis momentos de descontração. A Antônio Tadeu Gomes agradeço pela sua disponibilidade para esclarecimentos sobre seus trabalhos anteriores e pelas contribuições que ajudaram a definir os rumos da minha pesquisa.

Aos meus padrinhos Rômulo e Elizete Siqueira, obrigado pela acolhida no meu primeiro mês de curso, pois, quando várias eram as minhas incertezas, foram o meu porto seguro.

Agradeço também aos professores e funcionários da PUC-Rio que, de alguma forma, colaboraram no decorrer deste trabalho, e que fazem dessa instituição um centro de excelência reconhecido internacionalmente. Finalmente, agradeço à CAPES, pelo financiamento do meu trabalho a partir de seu programa de bolsas de mestrado, e à PUC-Rio, pela concessão da bolsa de isenção de taxas escolares. 


\section{Resumo}

Moreno, Marcelo Ferreira; Soares, Luiz Fernando Gomes. Um Framework para Provisão de QoS em Sistemas Operacionais. Rio de Janeiro, 2002. 105p. Dissertação de Mestrado - Departamento de Informática, Pontifícia Universidade Católica do Rio de Janeiro.

A demanda progressiva por aplicações multimídia distribuídas, caracterizadas por fortes exigências sobre os recursos computacionais, torna evidente a necessidade de provisão de qualidade de serviço (QoS) em cada um dos subsistemas envolvidos, como redes de comunicação e sistemas operacionais. Ao mesmo tempo, tais subsistemas devem ser flexíveis para que possam oferecer novos serviços a aplicações futuras, ou seja, devem ser adaptáveis em tempo de execução. Especificamente, sistemas operacionais de uso geral provêm pouco ou nenhum suporte a QoS e à adaptabilidade dos serviços, impulsionando vários estudos isolados nessas áreas. Observando-se algumas tecnologias implementadas em sistemas operacionais específicos, nota-se que os mecanismos de provisão possuem certas semelhanças funcionais. Assim, este trabalho propõe uma arquitetura adaptável para a provisão de QoS nos subsistemas de rede e de escalonamento de processos de sistemas operacionais, independente de implementação, através da descrição de frameworks genéricos. É demonstrado, também, como os pontos de flexibilização desses frameworks podem ser especializados para a implementação de alguns modelos de QoS. Por último, é proposto um cenário de uso da arquitetura, no qual um sistema operacional de uso geral ligeiramente modificado é utilizado como infra-estrutura para a instanciação dos frameworks de QoS.

\section{Palavras-chave}

Qualidade de Serviço, Sistemas Operacionais, Adaptabilidade, Frameworks, Controle de Admissão, Escalonamento de Recursos 


\section{Abstract}

Moreno, Marcelo Ferreira; Soares, Luiz Fernando Gomes. A Framework for QoS Provisioning in Operating Systems. Rio de Janeiro, 2002. 105p. MSc. Dissertation - Departamento de Informática, Pontifícia Universidade Católica do Rio de Janeiro

The progressive demand for distributed multimedia applications, which are characterized by strong requirements over computational resources, makes evident the need for quality of service (QoS) provisioning in each one of the involved subsystems (e.g. communication networks and operating systems). At the same time, these subsystems must be flexible enough that they can offer new services to future applications, or in other words, they must be adaptable at runtime. Specifically, general-purpose operating systems provide few or no QoS/service adaptability support, what have motivated many isolated studies about these topics. Observing some implemented technologies on specific operating systems, it is noted that the provisioning mechanisms have certain functional similarities. In this way, this work proposes an adaptable architecture for QoS provisioning on networking and process scheduling subsystems of operating systems, through the description of generic frameworks. It is demonstrated how the framework hot-spots can be specialized in order to implement some QoS models. Finally, it is proposed a scenario of use of the architecture, where a bit modified generalpurpose operating system is used as infrastructure for an instantiation of the QoS frameworks.

\section{Keywords}

Quality of Service, Operating Systems, Adaptability, Frameworks, Admission Control, Resource Scheduling 


\section{Sumário}

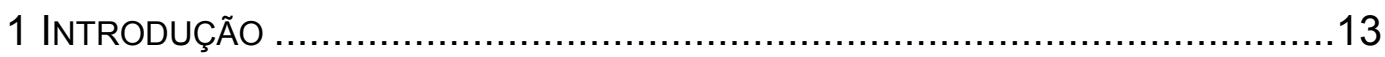

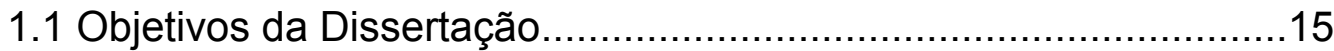

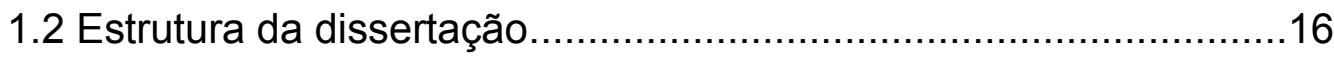

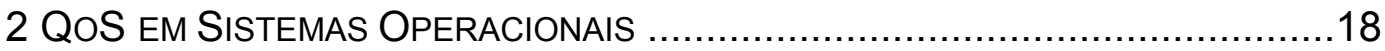

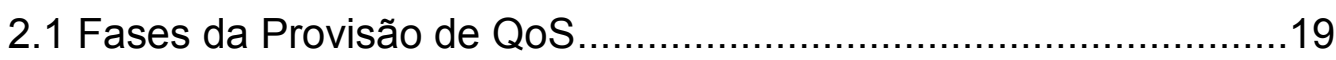

2.1.1 Iniciação do Provedor de Serviços ..............................................19

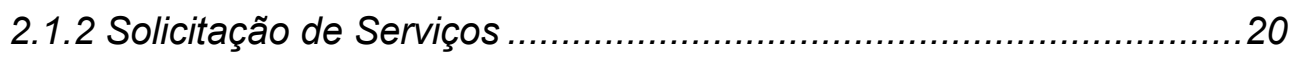

2.1.3 Estabelecimento de Contratos de Serviço........................................20

2.1.4 Manutenção de Contratos de Serviço ..............................................22

2.1.5 Encerramento de contratos de serviços .........................................23

2.2 Características relevantes de sistemas operacionais de uso geral 24

2.2.1 Arquitetura de sistemas operacionais...........................................24

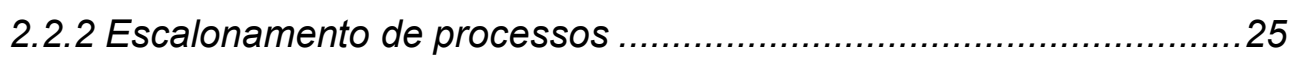

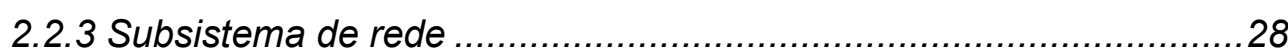

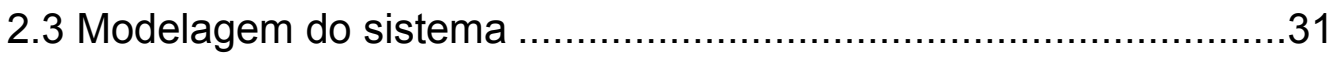

2.4 Escalonamento de processos com qualidade de serviço .................35

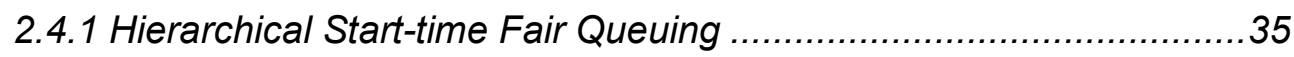

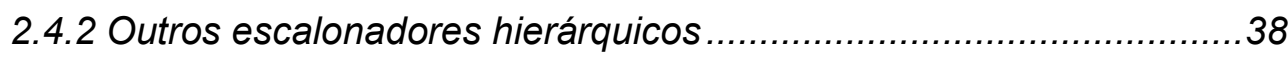

2.4.3 Meta-algoritmo para Políticas de Escalonamento..............................39

2.5 Subsistema de rede com qualidade de serviço ...............................41

2.5 .1 SUMO

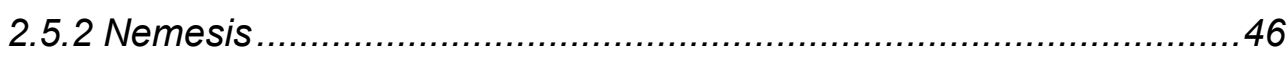

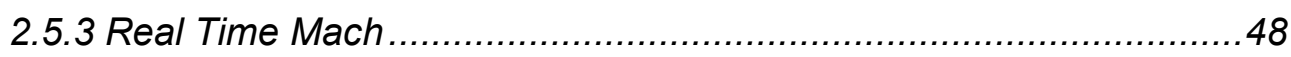

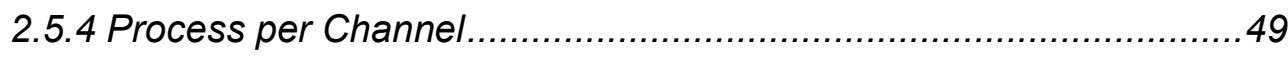

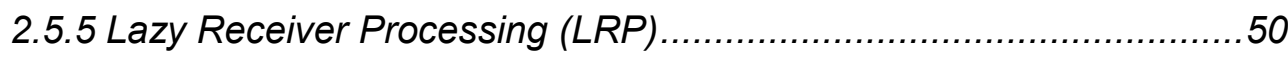

2.5.6 Linux Network Traffic Control (LinuxTC).......................................52

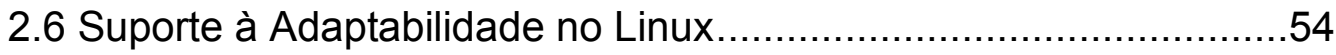

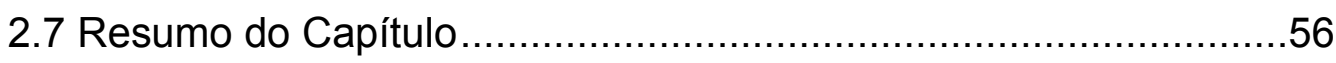

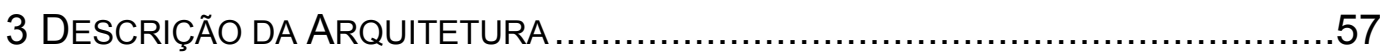

3.1 Parametrização de Serviços.........................................................59

3.1.1 Elementos do Framework para Parametrização de Serviços ............61 
3.1.2 Exemplo de Aplicação do Framework para Parametrização de Serviços.

3.2 Compartilhamento de Recursos .........................................63

3.2.1 Elementos do Framework para Escalonamento de Recursos ..........65

3.2.2 Exemplo de Aplicação do Framework para Escalonamento de

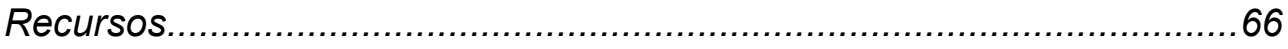

3.2.3 Elementos do Framework para Alocação de Recursos .....................68

3.2.4 Exemplo de Aplicação do Framework para Alocação de Recursos.. 69

3.3 Orquestração de Recursos.................................................71

3.3.1 Elementos do Framework para Negociação de QoS.......................74

3.3.2 Exemplo de Aplicação do Framework para Negociação de QoS......76

3.3.3 Elementos do Framework para Sintonização de QoS ......................79

3.3.4 Exemplo de Aplicação do Framework para Sintonização de QoS ....81

3.4 Adaptação de Serviços........................................................82

3.4.1 Elementos do Framework para Adaptação de Serviços ...................84

3.4.2 Exemplo de Aplicação do Framework para Adaptação de Serviços. 86

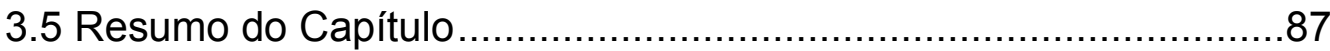

4 EXEMPLO dE APLICAÇÃO dA ARQUITETURA ......................................... 88

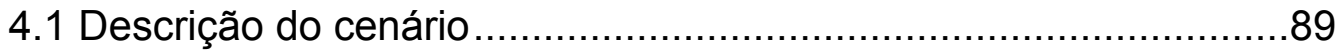

4.2 Infra-estrutura desenvolvida ..........................................90

4.3 Instanciação da arquitetura ....................................................94

4.3.1 Iniciação do sistema...........................................................94

4.3.2 Parametrização de serviços ......................................................96

4.3.3 Compartilhamento de recursos ...............................................97

4.3.4 Solicitação de serviços..............................................................100

4.3.5 Estabelecimento de contratos de serviço .....................................102

4.3.6 Manutenção de contratos de serviço..........................................103

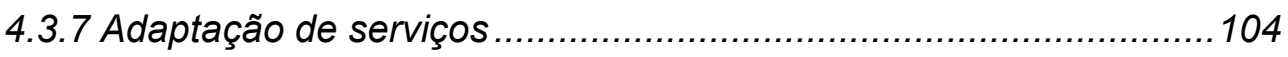

4.4 Resumo do Capítulo.........................................................105

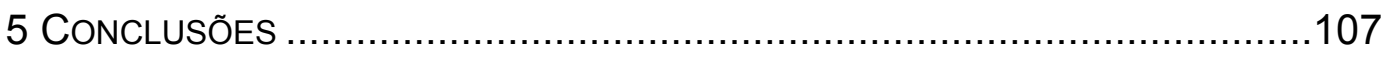

5.1 Contribuições da dissertação ................................................108

5.2 Trabalhos futuros ........................................................... 110

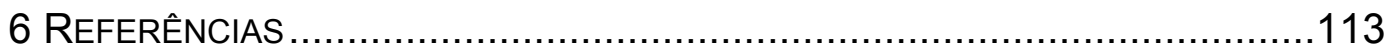




\section{Lista de Figuras}

Figura 2.1 - Visão geral do processamento de rede no padrão BSD4.4 .29

Figura 2.2 - Modelo baseado em redes de filas para sistemas operacionais em estações finais.

Figura 2.3 - Exemplo de estrutura de escalonamento ..........................36

Figura 2.4 - Interação do Gerenciador de QoS com o escalonador hierárquico

Figura 2.5 - Diagrama temporal de um ciclo de execução do algoritmo LDS.

Figura 2.6 - Gerenciador de fluxos proposto por (Campbell, 1996).........46

Figura 2.7 - Arquitetura do sistema Nemesis.....................................47

Figura 2.8 - Arquitetura de gerenciamento de recursos do Nemesis.......48

Figura 2.9 - Processamento dos dados de rede.................................52

Figura 2.10 - Modelo de disciplina de enfileiramento, definido em (Almesberger, 1999).

Figura 2.11 - Exemplo de hierarquia de disciplinas de enfileiramento, utilizando uma notação em árvore.

Figura 3.1 -Tipos de hot-spots de uma arquitetura modelada pelos frameworks genéricos de (Gomes, 1999)

Figura 3.2 - Framework para Parametrização de Serviços.

Figura 3.3 - Exemplo de aplicação do Framework para Parametrização de Serviços

Figura 3.4 - Exemplo de árvore de recursos virtuais para o recurso CPU64

Figura 3.5 - Framework para Escalonamento de Recursos. .65

Figura 3.6 - Exemplo de aplicação do Framework para Escalonamento de Recursos 67

Figura 3.7 - Framework para Alocação de Recursos 69

Figura 3.8 - Exemplo de aplicação do Framework para Alocação de Recursos .70

Figura 3.9 - Framework para Negociação de QoS .75

Figura 3.10 - Exemplo de aplicação do Framework para Negociação de QoS .77

Figura 3.11 - Framework para Sintonização de QoS .80

Figura 3.12 - Exemplo de aplicação do Framework para Sintonização de QoS

Figura 3.13 - Framework para Adaptação de Serviços. 
Figura 3.14 - Exemplo de aplicação do Framework para Adaptação de

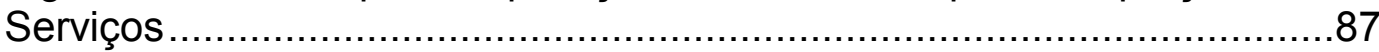

Figura 4.1 - Cenário de provisão de serviços Intserv .............................90

Figura 4.2 - Modelo de implementação de estratégias de admissão através de módulos em linguagem $C$

Figura 4.3 - Trecho de código de um controlador de admissão para o acesso aos módulos de estratégias de admissão ...................................93

Figura 4.4 - Visão geral da implementação ...........................................95

Figura 4.5 - Árvore de recursos virtuais da fila de pacotes........................96

Figura 4.6 - Aplicação do framework para Parametrização de Serviços ..97

Figura 4.7 - Aplicação do framework de escalonamento de recursos ......98

Figura 4.8 - Aplicação do framework para Alocação de Recursos .........100

Figura 4.9 - Aplicação do framework para Negociação de QoS .............102

Figura 4.10 - Aplicação do framework para Adaptação de Serviços ......105 


\section{Lista de Tabelas}

Tabela 2.1 - Descrição do processamento de rede no padrão BSD4.4 ....30

Tabela 2.2 - Cabeçalho da Tabela LDS................................................

Tabela 4.1 - Primitivas da API de solicitação de serviços.........................101 